\title{
Impacto da farinha de mandioca fortificada com ferro aminoácido quelato no nível de hemoglobina de pré-escolares ${ }^{1}$
}

\section{Impact of cassava flour fortified with iron amino acid chelate on the hemoglobin level in pre-schools}

Rahilda Brito TUMA ${ }^{2}$

Lucia Kiyoko Ozaki YUYAMA ${ }^{3}$

Jaime Paiva Lopes AGUIAR ${ }^{3}$

Hedylamar Oliveira MARQUES 4

RES U M O

\section{Objetivo}

Avaliou-se o impacto da farinha de mandioca fortificada com ferro aminoácido quelato em 80 pré-escolares de uma Unidade Filantrópica de Manaus, AM, distribuídos aleatoriamente em quatro grupos de 20 crianças cada, por um período de 120 dias.

\section{Métodos}

Foram utilizadas farinha de mandioca sem fortificação (Grupo zero) e fortificada com 1, 2 e 3mg de Fe/dia, correspondendo a quantias diárias de 5, 10 e $15 \mathrm{~g}$ de farinha, respectivamente, as quais foram distribuídas no horário do almoço, sendo ainda entregue às famílias a quantidade destinada ao consumo do final de semana. O estado nutricional das crianças foi avaliado no início e ao final do experimento, adotando-se como limite discriminatório entre eutrofia/desnutrição o ponto de corte <-2 escores-Z, de acordo com os critérios da Organização Mundial da Saúde, e estabelecendo-se como ponto de corte para a ocorrência de anemia ferropriva o teor de hemoglobina inferior a $11 \mathrm{~g} / \mathrm{dL}$.

\footnotetext{
1 Projeto financiado pelo CNPq proc.521950/95-3 e INPA:PPI 1-3100. Trabalho elaborado a partir da dissertação de mestrado de R.B. TUMA. "Fortificação da farinha de mandioca (Manihot esculenta, Crantz) com ferro aminoquelado impacto no nível de hemoglobina de pré-escolares", Manaus: UA, 2000. 106p.

${ }^{2}$ Universidade Federal do Pará. R. Augusto Corrêa, n.1, Caixa Postal 479, 66075-110, Belém, PA, Brasil. E-mail: ertuma@amazon.com.br

3 Instituto Nacional de Pesquisa da Amazônia, Coordenação de Pesquisas em Ciências da Saúde (INPA/CPCS). Av. André Araújo, n. 2936, Bairro Petrópolis, 69083-000, Manaus, AM, Brasil. Correspondência para/Correspondence to: L.K.O.YUYAMA. E-mail: yuyama@inpa.gov.br

4 Fundação de Hematologia e Hemoterapia do Amazonas (HEMOAM). Av. Constantino Neri, n.4397, Bairro Chapada, 69050-002, Manaus, AM, Brasil
} 


\section{Resultados}

Houve uma recuperação das crianças com desnutrição crônica ao final do estudo, e ocorreu um aumento significativo $(p<5 \%)$ dos valores de hemoglobina de todos os pré-escolares, independentemente da concentração de ferro, de 11,4 $\pm 0,9 \mathrm{~g} / \mathrm{dL}$ para $12,2 \pm 0,8 \mathrm{~g} / \mathrm{dL}$. As crianças anêmicas que receberam a farinha de mandioca fortificada com $2 \mathrm{mg}$ de Fe/dia foram plenamente recuperadas ao final da pesquisa, demonstrando um bom desempenho desse grupo em relação aos demais.

\section{Conclusão}

Sugere-se um estudo duplo cego para a consolidação da recomendação da farinha de mandioca fortificada com ferro na prevenção de anemia ferropriva em pré-escolares da região amazônica.

Termos de indexação: farinha, pré-escolar, anemia, agentes fortificantes de ferro, desnutrição, hemoglobinas.

\section{Objective}

The impact of the cassava flour fortified with iron amino acid chelate was evaluated in 80 pre-scholars of a Philanthropic Unit of Manaus, state of Amazonas, randomly distributed in four groups of 20 children each, for a period of 120 days.

\section{Methods}

Cassava flour was used without fortification (group zero) or fortified with 1, 2 and 3mg of Felday, corresponding respectively to 5, 10 and $15 \mathrm{~g}$ of flour/day, which were given to the children at lunch time on weekdays. The equivalent amount was previously distributed to their families for flour intake also during the weekends. In the beginning and at the end of the experiment the children's nutritional status was evaluated, being adopted the cutoff point <-2 Z-scores as a discriminating limit between eutrophy/ malnutrition, in agreement with the World Health Organization criteria, as well as being established as a cutoff point for the occurrence of iron deficiency anemia a hemoglobin rate of less than $11 \mathrm{~g} / \mathrm{dL}$.

\section{Results}

At the end of this study, children recovered from chronic malnutrition, and a significant increase $(p<5 \%)$ of the hemoglobin rates, independently of iron concentration, from $11.4 \pm 0.9 \mathrm{~g} / \mathrm{dL}$ to $12.2 \pm 0.8 \mathrm{~g} / \mathrm{dL}$, was observed in all individuals. The formerly anemic children who received cassava flour fortified with $2 \mathrm{mg}$ of Felday were fully recovered at the end of the research, demonstrating a good performance of this group in relation to the others.

\section{Conclusion}

A double-blind study is suggested for the consolidation of the recommendation of cassava flour fortified with iron in the prevention of iron deficiency anemia in pre-scholars of the Amazon region.

Index terms: flour, child, preschool, anemia, iron chelaling agents, nutrition disorders, hemoglobins. 


\section{N T R O D U ÇÃ O}

A anemia nutricional é definida pela Organização Mundial da Saúde (Organización..., 1968) como "um estado em que a concentração de hemoglobina do sangue é anormalmente baixa em conseqüência da carência de um ou mais nutrientes essenciais, qualquer que seja a origem dessa carência". Já a anemia por deficiência de ferro resulta de longo período de balanço negativo entre a quantidade de ferro biologicamente disponível e a necessidade orgânica desse oligoelemento.

Atualmente, a anemia por deficiência de ferro é a mais comum das carências nutricionais, atingindo cerca de um bilhão de pessoas no mundo todo, sendo mais prevalente em mulheres e crianças, principalmente nos países subdesenvolvidos. Constitui sério problema de saúde pública, pois pode resultar em prejuízos no desenvolvimento mental e psicomotor das crianças, aumento da morbimortalidade materna e infantil, queda no desempenho do indivíduo no trabalho e redução da resistência às infecções (Lonnerdal \& Dewey, 1996).

Considerando que a anemia é o último estágio da deficiência de ferro, calcula-se poder haver na população pelo menos o mesmo número de casos de deficiência sem instalação do quadro de anemia, ampliando, de forma contundente, a magnitude dessa doença em nível mundial (Morais \& Fagundes Neto, 1997).

Estudos epidemiológicos têm evidenciado considerável aumento da ocorrência desse distúrbio nutricional em indivíduos de todos os níveis socioeconômicos, ao contrário do que ocorre com a desnutrição, a qual vem apresentando tendência de declínio nas últimas décadas e cuja prevalência se concentra na população pobre (Leone, 1993).

Na Região Norte, em Manaus, merecem atenção os trabalhos com pré-escolares, na área ribeirinha dos rios Solimões e Negro, realizados por Giugliano et al. (1978) e Yuyama et al. (2000), que detectaram anemia ferropriva nos grupos estudados. A presença dessa doença pode ser explicada, em parte, pela monotonia alimentar, registrada no Estudo Nacional de Despesa Familiar (ENDEF), realizado entre 1974 e 1975 (Instituto Brasileiro..., 1977), no qual se observa um elenco de apenas sete alimentos (mandioca, trigo, arroz, açúcar, gordura, feijão e milho) respondendo por setenta e cinco por cento das calorias diárias ingeridas pela população amazônica (Carvalho, 1989). Ademais, o risco de anemia aumenta significativamente à medida que se reduz a ingestão de ferro (Monteiro et al., 2000).

Objetivando reduzir a ocorrência de anemia ferropriva, muitas estratégias têm sido propostas; entretanto, a fortificação da dieta com ferro é considerada por muitos pesquisadores e pela OMS como uma das melhores opções, pelo alcance de todos os segmentos populacionais, com possibilidade de utilização por longo período e sem necessidade de cooperação individual efetiva. Constitui, portanto, medida de baixo custo, rápida aplicação, alta efetividade e flexibilidade, sendo socialmente aceita, uma vez que não interfere no modelo alimentar da população, pois utiliza alimentos de uso corrente. Além disso, com as pequenas doses de fortificante adicionadas aos alimentos, os riscos de efeitos colaterais e toxicidade são mínimos (Fisberg et al., 1998). No Chile, os Guias Alimentares Baseados em Alimentos (GABA) representam uma boa alternativa para melhorar a adequação nutricional de dietas, e a fortificação de alimentos e a suplementação dirigida a grupos vulneráveis são práticas necessárias e complementares à implementação dos Guias (Oyarzún et al., 2001).

No Brasil, o uso de alimentos fortificados com ferro aminoácido quelato, como nos estudos utilizando leite em pó e fluído (Torres et al., 1996) e fórmulas lácteas (Fisberg, 1996), tem demonstrado bons resultados.

Quanto à escolha do alimento-veículo, considera-se que, no Brasil, a farinha de trigo, o fubá de milho e o açúcar seriam os mais indicados para uma ação de abrangência 
nacional. Porém, no caso das Regiões Norte e Nordeste, a farinha de mandioca, por apresentar alto consumo, poderia ter seu valor nutricional melhorado através da fortificação com ferro.

O grande volume de produção e consumo de farinha de mandioca na Região Norte e a ocorrência de anemia ferropriva na população suscitaram a necessidade de se avaliar o impacto da fortificação desse alimento com ferro em pré-escolares de uma Unidade Filantrópica de Manaus, AM.

\section{MATERIALE MÉTODOS}

O fortificante utilizado foi o ferro aminoácido quelato (Ferrochel Amino Acid Chelate) da Albion Lab. Inc, na concentração de $0,2 \mathrm{~g}$ de ferro/ $\mathrm{kg}$ de farinha de mandioca. A adição foi após a prensagem das raízes da mandioca, seguindo o processo de produção regional da farinha, ou seja, descasque, trituração, prensagem, fortificação, torrefação, peneiragem, classificação e embalagem do produto.

A análise química da farinha de mandioca foi realizada de acordo com as recomendações da Association of Official Analytical Chemists (AOAC) (1995), e a quantificação do teor de ferro foi determninada pelo processo de digestão via úmida, seguida de leitura em espectrofotômetro de absorção atômica modelo 1100 de acordo com as Normas do Instituto Adolfo Lutz (1985) e com o Manual da Perkin-Elmer (Perkin-Elmer, 1990). A análise microbiológica seguiu o método de Number Multiple Pipe (NMP) (Brasil..., 1995).

Por envolver a participação de seres humanos, a pesquisa foi submetida à aprovação do Comitê de Ética da Universidade do Amazonas e conduzida no sentido de coadunar-se com os princípios científicos e bioéticos.

Foi realizado um ensaio clínico randomizado, envolvendo 80 pré-escolares, selecionados entre as 300 crianças matriculadas na creche, na faixa etária de dois a seis anos, em blocos inteiramente casualizados assim distribuídos: Grupo 1: 20 crianças receberam $5 \mathrm{~g}$ de farinha fortificada contendo $1 \mathrm{mg}$ de Fe/dia; Grupo 2: 20 crianças receberam $10 \mathrm{~g}$ de farinha fortificada contendo $2 \mathrm{mg}$ de Fe/dia; Grupo 3: 20 crianças receberam $15 \mathrm{~g}$ de farinha fortificada contendo 3mg de Fe/dia; e Grupo 4: 20 crianças receberam $5 \mathrm{~g}$ de farinha sem fortificante. O período experimental foi de 120 dias e, diariamente, os pré-escolares recebiam na creche, de segunda a sexta-feira, no almoço, farinha de mandioca de acordo com o delineamento experimental. No final de semana e nos feriados, os pais se incumbiam de administrar a quantidade individualizada às crianças em casa no almoço. O controle em relação ao consumo das farinhas pelos pré-escolares ficou a cargo da pós-graduanda e dos técnicos do Laboratório de Nutrição e Físico-Química de Alimentos do Instituto Nacional de Pesquisas da Amazônia, que diariamente preparavam as porções de farinha de mandioca em sacos plásticos de $100 \mathrm{~g}$, identificavam-nas e distribuíam-nas às crianças, sempre no mesmo horário. A farinha de mandioca foi plenamente aceita pelos pré-escolares, pois ela já faz parte do hábito alimentar. No início do experimento foram excluídas as crianças cujos pais não assinaram o termo de consentimento e aquelas com anemia grave $(\mathrm{Hb}<9,5 \mathrm{~g} / \mathrm{dL}$ de sangue), e ao final do experimento, as que compareceram à creche por período inferior a $50 \%$ dos dias de intervenção e as que não participaram da avaliação final. As crianças com anemia grave foram encaminhadas ao setor médico da Entidade para tratamento, ou seja, suplementação com ferro, e não participaram do projeto.

A avaliação dietética das refeições consumidas pelas crianças na creche (desjejum, almoço e lanche) foi realizada por meio da aplicação dos métodos prospectivos de registro alimentar (Philippi et al., 1996) e análise de amostra em duplicata (Association..., 1995), 
além da investigação da qualidade protéica pelo Método de Cômputo Químico, da quantificação do ferro dietético e da verificação do ferro disponível na dieta consumida (Monsen et al., 1978).

O levantamento da situação socioeconômica foi obtido a partir de dados das fichas individuais, complementados por meio de entrevista realizada com os pais das crianças e/ou responsáveis por elas.

As medidas de peso e altura foram tomadas com base nas recomendações de Jellife (1968). Para a classificação nutricional foram utilizados os critérios propostos pela OMS (1995), adotando-se como limite discriminatório entre eutrofia/desnutrição o ponto de corte $<-2$ escores-Z para os indicadores altura/idade (desnutrição crônica) e peso/altura (desnutrição aguda). Considerou-se como padrão de referência o do National Center for Health Statistics (National Center..., 1977).

A avaliação hematológica para a quantificação de hemoglobina foi efetuada imediatamente após a colheita de sangue no antebraço, por punção venosa, utilizando-se o Counter automatizado SPKS da Fundação de Hemoterapia e Hematologia do Amazonas (HEMOAM). Na caracterização da anemia foi adotado o ponto de corte proposto pela OMS em 1968 para crianças de seis meses a seis anos, ou seja, valor de hemoglobina abaixo de $11 \mathrm{~g} / \mathrm{dL}$ de sangue (Organización Mundial..., 1968).

Utilizou-se o programa Epi Info, versão 6.04b (Organização Mundial..., 1997) para a compilação das informações, o que permitiu uma visualização da situação socioeconômica das famílias e do perfil antropométrico das crianças. Para os dados categorizados foi utilizado o Teste Exato de Fisher e para a avaliação do nível de associação entre duas variáveis, o Qui-quadrado. A comparação de médias foi feita pela Análise de Variância e teste de Tukey para os dados paramétricos e pela Análise de Kruskal-Wallis para os não paramétricos, todos com significância de $5 \%$, ou seja, a = 0,05 (Toledo \& Ovalle, 1981).

\section{RESULTADOSE DISCUSS ÃO}

Toda a farinha utilizada foi submetida à análise microbiológica e encontrava-se em condições próprias para o consumo humano. Da quantidade de ferro aminoácido quelato adicionado durante o processamento, em torno de $94 \%$ permaneceu no produto final, o que indica a viabilidade técnica da adoção de um processo de fortificação, com tecnologia simples, no próprio local de produção.

O fortificante não provocou mudanças nos caracteres organolépticos do produto, corroborando outros estudos em que o ferro aminoácido quelato foi utilizado para fortificar alimentos (Fisberg, 1996; Torres et al., 1996; lost et al., 1998). Com isso, a aceitabilidade da farinha de mandioca pelas crianças não sofreu modificação.

Os cardápios avaliados durante 11 dias apresentaram marcada monotonia, ausência de peixe e ovos de qualquer espécie e inexpressiva quantidade de frutos e hortaliças. Tais limitações decorreram dos tipos de produtos oriundos das doações e da restrição de recursos financeiros para os gastos com alimentação, aliados à falta de orientação técnica para garantir a utilização racional dos gêneros alimentícios disponíveis, com a inclusão de alimentos regionais e de safra, e para permitir a promoção de treinamento adequado ao pessoal envolvido no preparo das refeições.

Tendo como parâmetro a recomendação diária de calorias e nutrientes do National Research Council (NRC) (National..., 1989), foram analisadas as refeições (colação, almoço e lanche) oferecidas pela creche às crianças estudadas, durante 11 dias, obtendo-se uma adequação de 52\% para ferro, 60\% para energia $139 \%$ e para proteína (Tabela 1).

Em relação ao ferro dietético, foi observado que a maioria dos cardápios apresentou disponibilidade média estimada e quantidade média de ferro ingerido e absorvido de 5,18mg e 0,64mg, respectivamente (Tabela 2), 
de acordo com os valores preconizados por Monsen et al. (1978).

A deficiência de ferro na dieta tem sido relatada em diversos estudos envolvendo pré-escolares albergados em creches, como os de Mazzili (1987) e Sichieri et al. (1988). Segundo registros de Sigulem et al. (1985), mesmo com a elevação da renda familiar e o conseqüente aumento da ingestão desse

Tabela 1. Cobertura média das recomendações diárias de energia, proteína e ferro, por meio da dieta servida na creche.

\begin{tabular}{cccc}
\hline \multirow{2}{*}{ Cardápio } & \multicolumn{3}{c}{ Cobertura Média (\%) } \\
\cline { 2 - 4 } & Energia & Proteína & Ferro \\
\hline 1 & 51 & 125 & 38 \\
2 & 62 & 133 & 49 \\
3 & 67 & 152 & 56 \\
4 & 68 & 122 & 59 \\
5 & 58 & 91 & 24 \\
6 & 52 & 124 & 49 \\
7 & 51 & 121 & 64 \\
8 & 52 & 167 & 70 \\
9 & 76 & 178 & 48 \\
10 & 67 & 188 & 73 \\
11 & 57 & 134 & 40 \\
\hline$\overline{\mathbf{X}}$ & 60 & 139 & 52 \\
\hline
\end{tabular}

$\overline{\mathbf{X}}=$ Média. mineral, a dieta consumida não atendia a necessidade diária dos pré-escolares. Como a presente pesquisa a adequação desse nutriente correspondeu a $52 \%$ do valor recomendado pelo NRC (National..., 1989), é possível deduzir que, mesmo com o acréscimo do ferro oriundo das refeições domiciliares, a recomendação não seria alcançada.

A distribuição energética percentual dos macronutrientes da dieta apresentou-se equilibrada (Tabela 3); porém, a quantidade média de proteína consumida $(33 \pm 7 \mathrm{~g} / \mathrm{dia})$ ultrapassou em 39\% a recomendação diária, remetendo à hipótese de que parte das proteínas esteja sendo desviada da função plástica para o fornecimento de energia.

Apesar do excesso protéico, a análise da composição aminoacídica (Tabela 4) indicou o triptofano como aminoácido limitante da dieta (Cômputo Químico $=0,45$ ). O déficit de triptofano, precursor da serotonina, pode gerar desequilíbrio no pool orgânico de aminoácidos e, a longo prazo, resultar em alterações orgânicas como retardo no crescimento físico e no desenvolvimento mental e redução na capacidade de aprendizagem e compreensão das crianças (Veloso, 1998).

Tabela 2. Disponibilidade e nível estimado de absorção do ferro dos cardápios.

\begin{tabular}{|c|c|c|c|c|c|c|c|c|c|}
\hline \multirow{2}{*}{ Cardápio } & \multirow{2}{*}{ Carne (g) } & \multirow{2}{*}{$\begin{array}{c}\text { Ácido } \\
\text { ascórbico } \\
\text { (mg) }\end{array}$} & \multirow{2}{*}{$\begin{array}{c}\text { Classificação } \\
\text { Dieta }\end{array}$} & \multicolumn{3}{|c|}{ Ferro Ingerido (mg) } & \multicolumn{3}{|c|}{ Ferro Absorvido (mg) } \\
\hline & & & & Heme & $\tilde{N}$ Heme & Total & Heme & $\tilde{N}$ Heme & Total \\
\hline 1 & 43,30 & 31,13 & MD & 1,53 & 2,31 & 3,84 & 0,35 & 0,12 & 0,47 \\
\hline 2 & 60,00 & 43,76 & $M D$ & 1,96 & 2,93 & 4,89 & 0,45 & 0,15 & 0,60 \\
\hline 3 & 40,00 & 41,78 & $M D$ & 2,23 & 3,35 & 5,58 & 0,51 & 0,17 & 0,68 \\
\hline 4 & 40,00 & 25,47 & MD & 2,38 & 3,57 & 5,95 & 0,55 & 0,18 & 0,73 \\
\hline 5 & 30,00 & 41,33 & $M D$ & 0,97 & 1,45 & 2,42 & 0,22 & 0,07 & 0,29 \\
\hline 6 & 40,00 & 46,94 & $M D$ & 1,95 & 2,92 & 4,87 & 0,45 & 0,15 & 0,60 \\
\hline 7 & 50,00 & 91,78 & MD & 2,58 & 3,88 & 6,46 & 0,59 & 0,19 & 0,78 \\
\hline 8 & 80,00 & 36,79 & $M D$ & 2,79 & 4,18 & 6,97 & 0,64 & 0,21 & 0,85 \\
\hline 9 & 100,00 & 27,68 & $A D$ & 1,90 & 2,86 & 4,76 & 0,44 & 0,23 & 0,67 \\
\hline 10 & 100,00 & 28,51 & $A D$ & 2,92 & 4,37 & 7,29 & 0,67 & 0,22 & 0,89 \\
\hline 11 & 100,00 & 34,59 & $A D$ & 1,59 & 2,39 & 3,98 & 0,37 & 0,12 & 0,49 \\
\hline$\overline{\mathbf{X}}$ & 62,20 & 33,80 & - & 2,07 & 3,11 & 5,18 & 0,48 & 0,16 & 0,64 \\
\hline SD & 27,62 & 21,48 & - & 0,58 & 0,87 & 1,48 & 0,13 & 0,05 & 0,18 \\
\hline
\end{tabular}

Fonte: Monsen et al. (1978); $\tilde{N}=$ Não; $\bar{X}=$ Média Disponibilidade; AD = Alta Disponibilidade; SD = Desvio-padrão. 
Tabela 3. Distribuição energética dos macronutrientes da dieta servida na creche.

\begin{tabular}{|c|c|c|c|c|}
\hline \multirow{2}{*}{ Cardápio } & \multirow{2}{*}{ Energia Total (kcal) } & \multicolumn{3}{|c|}{ Distribuição energética dos nutrientes (\%) } \\
\hline & & Proteína & Carboidrato & Lipídio \\
\hline 1 & 770 & 15 & 60 & 25 \\
\hline 2 & 940 & 13 & 69 & 18 \\
\hline 3 & 1132 & 13 & 70 & 17 \\
\hline 4 & 1041 & 11 & 70 & 19 \\
\hline 5 & 882 & 10 & 69 & 21 \\
\hline 6 & 798 & 14 & 65 & 21 \\
\hline 7 & 769 & 15 & 68 & 17 \\
\hline 8 & 793 & 20 & 60 & 20 \\
\hline 9 & 1149 & 15 & 65 & 20 \\
\hline 10 & 1026 & 18 & 58 & 24 \\
\hline 11 & 860 & 15 & 67 & 18 \\
\hline$\overline{\mathbf{X}}$ & 924 & 14 & 66 & 20 \\
\hline SD & 143 & 3 & 4 & 2 \\
\hline
\end{tabular}

$\overline{\mathbf{X}}=$ Média; SD = Desvio-padrão.

Tabela 4. Cômputo químico da dieta comparado ao padrão FAO/OMS.

\begin{tabular}{lccc}
\hline \multirow{2}{*}{ Aminoácidos } & \multicolumn{2}{c}{$\begin{array}{c}\text { Quantidade } \\
\text { (g/g de proteína) }\end{array}$} & \multirow{2}{*}{$\begin{array}{c}\text { Cômputo } \\
\text { Químico }\end{array}$} \\
\cline { 2 - 3 } FAO/OMS & Dieta & \\
\hline Isoleucina & 2,8 & 3,2 & 1,14 \\
Leucina & 6,6 & 5,4 & 0,82 \\
Lisina & 5,8 & 6,4 & 1,10 \\
Metionina+ cistina & 2,5 & 3,3 & 1,32 \\
Histidina & 1,9 & 5,9 & 3,10 \\
Fenilalanina + tirosina & 6,3 & 5,5 & 0,87 \\
Treonina & 3,4 & 4,0 & 1,18 \\
Triptofano & 1,1 & 0,5 & $\left.0,45^{*}\right)$ \\
Valina & 3,5 & 3,5 & 1,0 \\
\hline
\end{tabular}

${ }^{(*)}$ aminoácido limitante da dieta.

O perfil de consumo alimentar caracterizado por baixa ingestão energético e elevada ingestão protéica reflete ainda uma situação observada no passado, em pesquisas realizadas em Manaus.

Em relação à composição do grupo estudado, das 80 crianças do estudo inicial, cinco foram eliminadas por desligamento espontâneo da creche ou por não terem sido submetidas à avaliação final. A perda amostral foi, portanto, de $6 \%$, podendo ser considerada baixa, se comparada com estudos semelhantes realizados por autores como Nogueira et al.
(1992), os quais, em ensaio utilizando biscoito fortificado em uma creche no Piauí, tiveram uma perda de $46,6 \%$ da amostra em 90 dias. Fisberg, (1996), testando uma fórmula láctea em duas creches em São Paulo, tiveram uma perda amostral de 18,4\% em 180 dias, e Vítolo et al. (1998), utilizando cereal fortificado em pré-escolares, perderam $22 \%$ da amostra inicial ao longo de 60 dias. A freqüência média foi de 102 dias, correspondendo a $85 \%$ dos dias previstos no projeto.

A distribuição das crianças nos quatro grupos foi homogênea em relação ao sexo e à renda familiar, que variou entre $R \$ 291,60$ e $\mathrm{R} \$ 441,90$. A maioria delas (80\%) provém de famílias de baixa renda, compostas por dois a cinco membros, acompanhando a tendência nacional dos últimos anos. Verificou-se a predominância de grupos mononucleares contando com a presença do pai e da mãe. As condições socioeconômicas e ambientais das famílias entrevistadas também não diferiram significativamente entre si.

A pequena variabilidade de valores da renda familiar não permite levantar a significância estatística do seu impacto sobre os parâmetros avaliados neste estudo. Além 
disso, em pesquisas realizadas por Szarfarc et al. (1988), Romani et al. (1991) e Monteiro et al. (2000) foi demonstrado que a elevação da renda não garantiu a correção da anemia com a mesma eficácia com que promoveu a redução da desnutrição energético-protéica.

Quanto aos parâmetros antropométricos, na avaliação inicial apenas $1,3 \%$ e $2,7 \%$ das crianças apresentavam déficit na relação peso/altura e altura/idade, respectivamente, caracterizando o quadro de desnutrição aguda e crônica (Tabela 5). Ao final do estudo, houve a recuperação das crianças com desnutrição crônica.

De acordo com Morais \& Fagundes Neto (1997) a melhoria do estado nutricional se deve provavelmente à reversão das anormalidades intestinais durante a ferroterapia e à conseqüente melhoria no aproveitamento dos alimentos consumidos. Já Morais et al. (1993) e Lawless et al. (1994) entendem que esse efeito seja resultante da redução da anorexia, geralmente presente nos casos de anemia.

Entretanto, neste estudo, em relação ao grupo placebo, outra possível explicação para a melhoria do estado nutricional seria o chamado Efeito Reverso (Bowling, 1997). A simples presença do pesquisador, mesmo tomando todas as providências para não interferir nas atividades da creche, pode ter alterado algumas rotinas, ocasionando maior atenção às crianças, melhoria do processo de confecção e distribuição das refeições e maiores cuidados de higiene geral. Esse fenômeno é freqüentemente observado em estudos que envolvem variáveis sociais e contato prolongado entre pesquisador e pesquisados.

No tocante à anemia ferropriva, no início deste trabalho $23,0 \%$ dos pré-escolares encontravam-se anêmicos, percentual próximo dos obtidos em pesquisas semelhantes realizadas por outros autores: Vítolo et al. (1998)

Tabela 5. Comparação entre as proporções de altura por idade (A/I) em escore-Z, peso por idade (P/I) em escore-Z, peso por altura (P/A) e hemoglobina em relação a antes e depois do tratamento.

\begin{tabular}{|c|c|c|c|c|c|c|}
\hline \multirow{2}{*}{ Variáveis } & \multicolumn{2}{|c|}{ Antes } & \multicolumn{2}{|c|}{ Depois } & \multirow[b]{2}{*}{ Total } & \multirow[b]{2}{*}{ p-valor } \\
\hline & $n$ & $\%$ & $n$ & $\%$ & & \\
\hline$A / l<-2$ escore $Z$ & 2 & 2,7 & 0 & 0 & 75 & - \\
\hline $\mathrm{P} / \mathrm{I}<-2$ escore $\mathrm{Z}$ & 2 & 2,7 & 1 & 1,3 & 75 & 0,9887 \\
\hline P/A $<-2$ escore $Z$ & 1 & 1,3 & 1 & 1,3 & 75 & 0,9986 \\
\hline $\mathrm{Hb}<11 \mathrm{~g} / \mathrm{dL}$ & 17 & 22,7 & 6 & 8,0 & 75 & 0,0127 \\
\hline
\end{tabular}

$\mathrm{Hb}=$ Hemoglobina

Tabela 6.Número de crianças anêmicas e média de hemoglobina antes e após a intervenção e diferença de hemoglobina (final menos a inicial).

\begin{tabular}{|c|c|c|c|c|c|c|c|c|c|c|c|}
\hline \multirow[t]{3}{*}{ Grupos } & \multicolumn{4}{|c|}{$\mathrm{Hb}<11 \mathrm{~g} / \mathrm{dL}$} & \multicolumn{6}{|c|}{ Média $\mathrm{Hb}(\mathrm{g} / \mathrm{dL})$} & \multirow[t]{3}{*}{ Diferença $\mathrm{Hb}$} \\
\hline & \multicolumn{2}{|c|}{ Antes } & \multicolumn{2}{|c|}{ Após } & \multicolumn{3}{|c|}{ Antes } & \multicolumn{3}{|c|}{ Após } & \\
\hline & $\mathrm{n}$ & $\%$ & $\mathrm{n}$ & $\%$ & $\mathrm{n}$ & & $\%$ & $n$ & & $\%$ & \\
\hline G1 & 5 & 25 & 3 & 15 & 11,27 & \pm & 0,98 & 11,98 & \pm & $0,91^{a}$ & $0,71^{a}$ \\
\hline $\mathrm{G} 2$ & 4 & 21 & 0 & 0 & 11,37 & \pm & 0,70 & 12,18 & \pm & $0,63^{a}$ & $0,81^{a}$ \\
\hline G3 & 6 & 32 & 3 & 16 & 11,48 & \pm & 0,90 & 12,26 & \pm & $0,92^{a}$ & $0,78^{a}$ \\
\hline $\mathrm{G} 4$ & 2 & 12 & 0 & 0 & 11,68 & \pm & 0,57 & 12,06 & \pm & $0,42^{a}$ & $0,38^{a}$ \\
\hline Total & 17 & 21 & 6 & 7 & 11,40 & \pm & 0,90 & 12,20 & \pm & $0,80^{*}$ & \\
\hline
\end{tabular}

Médias seguidas por letras distintas diferem entre si ao nível de $5 \%$ de significância, pelo teste de Tukey.

* pelo Teste de Kruskal-Wallis, p-valor mostra diferença estatisticamente significativa ao nível de $5 \%$, em relação as médias antes e depois do tratamento.

$\mathrm{G} 1$ = ferro 1mg; $\mathrm{G} 2$ = ferro $2 \mathrm{mg} ; \mathrm{G} 3$ = ferro 3mg; $\mathrm{G} 4$ = ferro sem fortificante; $\mathrm{Hb}=$ hemoglobina. 
registraram 20,8\% em São Paulo, Assis et al. (1997) encontraram 22,2\% na Bahia, Silva \& Sturion (1998) relataram 22,5\% em Piracicaba; tal semelhança de resultados provavelmente ocorreu por tratarem-se de amostras com condições socioeconômicas, de saúde e de nutrição similares ao do grupo estudado.

As crianças avaliadas neste trabalho, independentemente do teor de ferro ingerido por meio da farinha de mandioca, apresentaram aumento significativo da concentração de hemoglobina ao final do estudo (Tabela 5). No entanto, quando se analisa a concentração média final e o ganho nos diferentes grupos (Tabela 6), não há diferença significativa $(p<5 \%)$. Contudo, os pré-escolares que receberam a farinha de mandioca fortificada na concentração de $2 \mathrm{mg}$ de ferro apresentaram o melhor desempenho, com uma recuperação total das crianças anêmicas (Tabela 6). 0 mesmo não foi observado com o grupo que recebeu $3 \mathrm{mg}$ de ferro, provavelmente em virtude da freqüência do consumo entre as anêmicas, a qual oscilou em torno de $65 \%$.

O aumento da concentração de hemoglobina das crianças que não receberam a farinha fortificada pode estar relacionado ao tipo de alimentação servida na creche, a qual, de acordo com a avaliação sugerida por Monsen et al. (1978), apresentou nível médio de biodisponibilidade estimada de ferro e correspondeu à cobertura de aproximadamente 50\% da recomendação diária para pré-escolares.

Ratifica-se o importante papel das unidades de atendimento infantil direcionadas às famílias de baixo nível socioeconômico, especialmente no meio urbano, onde a participação da mãe no mercado de trabalho é fundamental na composição da renda familiar, conforme relatam Silva \& Sturion (1998). Entretanto, ressalta-se a possibilidade de o efeito reverso ter propiciado a melhoria do estado nutricional dos pré-escolares avaliados.
Estudos realizados por diferentes autores, utilizando ferro aminoácido quelato como fortificante de alimentos consumidos por crianças e adolescentes, e alguns ensaios clínicos, envolvendo tratamento de adultos com o mesmo produto, também constataram a elevação da concentração de hemoglobina, demonstrando a efetividade da fortificação (Pineda,1997; lost et al., 1998).

\section{O N CLUS Ã O}

Nas condições desta pesquisa, os resultados obtidos com a utilização da farinha de mandioca fortificada com ferro aminoácido quelato possibilitaram as seguintes conclusões: é viável fortificar a farinha de mandioca, por meio de processamento tecnológico simples, sem alterar o sistema regional adotado para produzi-la; o fortificante não provocou mudanças nos caracteres organolépticos da farinha de mandioca; a baixa perda amostral e a elevada freqüência média indicaram bom nível de adesão dos participantes; o impacto da utilização da farinha de mandioca fortificada com ferro aminoácido quelato sobre os níveis séricos de hemoglobina dos pré-escolares foi efetiva em todas as concentrações utilizadas, sendo a recuperação total de crianças anêmicas e o melhor desempenho constatados no grupo que recebeu $2 \mathrm{mg}$ de $\mathrm{Fe} /$ dia; a fortificação de alimentos básicos, de consumo maciço, como a farinha de mandioca na Região Norte, é uma alternativa promissora na prevenção de anemia ferropriva, necessitando de estudos duplo cego para a consolidação da recomendação.

\section{A GRADECIMENTOS}

Unidade Filantrópica (Casa da Criança) de Manaus, Fábrica Caiari Agroindústria Ltda, Albion Lab. INC, aos técnicos: Aiub Atem Dantas, Lucimar Siqueira, Maria Helena A. Cortez, ao Dr. Raul Guerra Queiroz pela revisão do Abstract e a Dra. Suely Costa pela revisão das análises estatísticas. 


\section{REFERÊ NCIAS BIBLIOGRÁFICAS}

ASSOCIATION OF OFFICIAL ANALYTICAL CHEMISTS. Official Methods of Analysis. 17.ed. Washington DC: [s.n.], 1995.

ASSIS, A.M, SANTOS, L.M.P., MARTINS, M.C., ARAÚJO, M.P.N., AMORIN, D.Q., MORRIS, S.S. Distribuição da anemia em pré-escolares do Semi-árido da Bahia. Cadernos de Saúde Pública, Rio de Janeiro, v.13, n.2, p.237-243, 1997.

BOWLING, A. Research methods in health buckingham. Philadelphia : Open University Press, 1997.

BRASIL. Ministério da Educação. Fundação de Assistência ao Estudante (MEC/FAE). Portaria MEC/FAE 174/95. Brasília, 1995.

CARVALHO, L.E. Nutrição e desnutrição na Amazônia: dos mitos à dura realidade. Boletim SBCTA, v.23, n.1/2, p.1-11, 1989.

FISBERG, M. Utilização de suplemento alimentar enriquecido com ferro, na prevenção de anemia em pré-escolares. Revista de Pediatria Moderna, Rio de Janeiro, v.32, n.7, p.753-57, 1996.

FISBERG, M., BRAGA, J.A.P., GIORGINI, E., PAULA, R.C. Tratamento e prevenção de anemia carencial por deficiência de ferro. Revista de Pediatria Moderna, Rio de Janeiro, v.34, n.10, p.651-657, 1998.

GIUGLIANO, R., SHRIMPTON, R., ARKCOLL, D.B., GIUGLIANO, L.G. PETRERE JR, M. Diagnóstico da realidade alimentar e nutricional do Estado do Amazonas, 1978. Acta Amazônica, n.2, p.47-48, 1978.

INSTITUTO ADOLFO LUTZ. Normas analíticas do Instituto Adolfo Lutz: análise de água e alimentos. 3.ed. São Paulo, 1985. v.1.

INSTITUTO BRASILEIRO DE GEOGRAFIA E ESTATÍSTICA. Estudo Nacional de Despesa Familiar: dados preliminares, consumo alimentar, antropometria. 4.ed. Rio de Janeiro, 1977. v.1

IOST, C., NAME, J.J., JEPPSEN, R.B., ASHMEAD, DeW. Repleting hemoglobin in iron deficiency anemia in young Children through liquid milk fortification with bioavailable iron amino acid chelate. J Am Coll Nutr, v.17, n.2, p.187-194, 1998.
JELLIFE, D.B. Evaluación del estado de nutrición de la comunidade com especial referencia a las encuestas en las regiones in desarrollo. Genebra: OMS, 1968.

LAWLESS, J.L., LATHAM, M.C., STEPHESON, L.S., KINOTI, S.N., PERTET, A.M. Iron suplementation improves appetite and growth in anemic Kenyan primary school children. Journal of Nutrition, v.124, p.645-654, 1994.

LEONE, C. Anemias Nutricionais. In: CONGRESSO BRASILEIRO DE PEDIATRIA, 28., 1993, Salvador. Anais... Salvador : [s.n.], 1993. p.2-8.

LONNERDALL, B., DEWEY, K.G. Epidemiologia da deficiência de ferro no lactante e na criança. Anais Nestlé, São Paulo, n.52, p.1-10, 1996.

MAZZILI, R.N. Valor nutricional da merenda escolar e sua contribuição para as recomendações nutricionais do pré-escolar matriculado em CEAP. Revista de Saúde Pública, São Paulo, v.21, p.246-254, 1987.

MONSEN, E.R, HALLBERG, L., LAYRISSE, M.G., HEGSTED, M., COOK, J.D., MERTZ, W., FINCH. C.A. Estimation of available dietary iron. American Journal of Clinical Nutrition, Bethesda, v.1, p.134-141, 1978.

MONTEIRO, C.A., SZARFARC, S.C., MONDINI, L. Tendência secular da anemia na infância na cidade de São Paulo (1984-1996). Revista Saúde Púlica, São Paulo, v.34, n.6, p.62-72, 2000. Suplemento.

MORAIS, M.B., FERRARI, A.A., FISBERG, M. Effect of oral iron therapy on physical growth. Revista Paulista de Medicina, São Paulo, v.111, n.6, p.439-444, 1993.

MORAIS, M.B., FAGUNDES NETO, U. Interação entre o intestino e a deficiência de ferro. Revista de Pediatria Moderna, Rio de Janeiro, v.33, n.8, p.589-597, 1997.

NATIONAL CENTER FOR HEALTH STATISTICS. Growth curves children birth-18. Washington DC : Printinc Office, 1977.

NATIONAL RESEARCH CUNCIL (USA). Recommended dietary allowances. 10.ed. Washington DC: National Academy Press, 1989.

NOGUEIRA, N.N., COLLI, C., COZZOLINO, S.M.F. Controle da anemia ferropriva em pré-escolares por 
meio da fortificação de alimento com concentrado de hemoglobina bovina (estudo preliminar). Cadernos de Saúde Pública, Rio de Janeiro, v.8, n.4, p.459-465, 1992.

ORGANIZACIÓN MUNDIAL DE LA SALUD. Anemias nutricionales. Ginebra, 1968. (Informe Técnico, n.405).

ORGANIZACIÓN MUNDIAL DE LA SALUD. La situación sanitaria mundial: informe sobre la salud em el mundo, 1995: reducir las desigualdades. Foro Mundial de la Salud, v.16, n.4, p.430-440, 1995.

ORGANIZAÇÃO MUNDIAL DA SAÚDE. Programa Epi Info: Versão 6.04 b. [s.l : s.n.], 1997.

OYARZÚN, M.T., YAUY, R., OLIVARES, S. Enfoque alimentario para mejorar la adecuación nutricional de vitaminas y minerales. Archivos Latinoamericanos de Nutrición, Guatemala, v.51, n.1, p.7-18, 2001.

PERKIN-ELMER. Analytical methods for atomic absorption spectrophotometry. Normalk (USA) : [s.n], 1990. p.BC-2.

PHILIPPI, S.T., SZARFARC, S.C., LATERZA, A.R. Virtual Nutri [Programa de Computador]. Versão 1.0 for Windows. [s.l : s.n.], 1996.

PINEDA, O. Clinical studies using Iron aminoacidchelate. Guatemala : Celanem, 1997.

ROMANI, S.A.M., LIRA, P.I.C., BATISTA FILHO, M., SEQUEIRA, L.A. S., FREITAS, C.L.C. Anemias em pré-escolares: diagnóstico, tratamento e avaliação/ Recife-PE-Brasil. Archivos Latinoamericanos de Nutrición, Guatemala, v.41, n.2, p.159-167, 1991.

SICHIERI, R., SZARFAC, S.C., MONTEIRO, C.A. Relação entre dieta e ocorrência de anemia ferropriva em crianças. Journal de Pediatria, v.64, n.5, p.169-174, 1988.

SIGULEM, D. M., TUDISCO, E.S., PAIVA, E.R., GUERRA, C.C.C. Anemia nutricional e parasitose intestinal em menores de 5 Anos. Revista Paulista de Medicina, São Paulo, v.103, n.6, p.308-312, 1985.
SILVA, M.V., STURION, G.L. Freqüência à creche e outros condicionantes do estado nutricional infantil. Revista de Nutrição, Campinas, v.11, n.1, p.58-68, 1998.

SZARFAC, S.C., MONTEIRO, C.A., MEYER, M., TUDISCO, E.S., REIS, I.M. Estudo das condições de saúde das crianças do município de São Paulo, 1984/1985: consumo alimentar. Revista de Saúde Pública, São Paulo, v.22, p.266-272, 1988.

TOLEDO, G.L., OVALLE, I.I. Estatística básica. São Paulo : Atlas, 1981.

TORRES, M.A.A., LOBO, N.F., SATO, K., SOUZA QUERIOS S. Fortificação do leite fluído na prevenção e tratamento da anemia carencial em crianças menores de 4 anos. Revista de Saúde Pública, São Paulo, v.30, n.4, p.350-357, 1996.

VELOSO, R.V. Recuperação do estado nutricional de ratos desnutridos: avaliação do emprego de uma mistura de alimentos não convencionais. Mato Grosso do Sul, 1998. Dissertação (Mestrado) - Universidade Federal do Mato Grosso, 1998.

VITOLO, M.R., AGUIRRE, A.N.C., KONDO, M.R., GIULIANO, Y., FERREIRA, NL. LOPEZ F.A. Impacto do uso de cereal adicionado de Fe sobre os níveis de $\mathrm{Hb}$ e antropometria de pré-escolares. Revista de Nutrição, Campinas, v.11, n.2, p.163-171, 1998.

YUYAMA, L.K.O., NAGAHAMA, D., RODRIGUES, M.L.C.F., ALENCAR, F.H.I., MARQUES, E.O., CORDEIRO, G.W.O. Estado nutricional e anemia ferropriva em pré-escolares de uma Unidade Filantrópica de Manaus AM. In: OBESIDADE E ANEMIA NA ADOLESCÊNCIA, 2000, Salvador. Anais... Salvador, 2000. p.209-210.

Recebido para publicação em 10 de abril de 2001 e aceito em 7 de fevereiro de 2002. 